\title{
Liquefied Medicinal Gas Dosage Form
}

National Cancer Institute

\section{Source}

National Cancer Institute. Liquefied Medicinal Gas Dosage Form. NCI Thesaurus. Code C149658.

A gas packaged under pressure, which is partially liquid (gas over liquid) at -50 degrees C. 